\title{
Vie et émotions des équipes entrepreneuriales étudiantes
}

\author{
STEPHANE FOLIARD \\ IUT Roanne, Université Jean Monnet - COACTIS, stephane.foliard@univ-st-stienne.fr \\ SANDRINE LE PONTOIS \\ IUT Roanne, Université Grenoble Alpes - CERAG, sandrine.lepontois@univ-st-stienne.fr
}

\section{Résumé}

Les programmes d'enseignement de l'entrepreneuriat font régulièrement appel au travail en équipe. Sur la durée, le niveau de motivation des étudiants est variable et des tensions apparaissent régulièrement. À partir d'une étude qualitative portant sur 13 équipes et 66 étudiants issus de deux écoles de commerce françaises, nous identifions 5 événements clés du processus entrepreneurial et les relions aux émotions positives et négatives qu'ils génèrent ainsi qu'à leur impact sur la motivation des membres de l'équipe et les stratégies de coping mobilisées. Notre contribution consiste à nommer ces émotions et à caractériser leur valence. Percevoir ces émotions et leurs effets permet d'identifier les équipes dysfonctionnelles ou d'entretenir les dynamiques positives par un accompagnement adapté.

\begin{abstract}
Entrepreneurship curricula regularly involve teamwork. Over time, the level of student motivation varies and tensions regularly arise. Based on a qualitative study of 13 teams and 66 students from two French business schools, we identify 5 key events in the entrepreneurial process and relate them to the positive and negative emotions they generate and their impact on the motivation of team members and the coping strategies mobilized. Our contribution is to name these emotions and characterize their valence. Perceiving these emotions allows us to identify dysfunctional teams or to maintain positive dynamics and to adapt the coaching.
\end{abstract}

\section{Mots-clés}

Equipe entrepreneuriale étudiante, événements, émotions, motivation, stratégies de coping.

\section{Key words}

Student entrepreneurial team, events, emotions, motivation, coping strategies. 


\section{Introduction}

L'éducation en entrepreneuriat fait de plus en plus appel à des méthodes actives. Elles reposent sur un apprentissage par la pratique incluant des contacts plus ou moins importants avec le monde réel et ses parties prenantes. Devant l'ampleur de la tâche (simuler la création ou créer réellement une entreprise) et la nécessité d'accompagner les étudiants dans ces démarches complexes, la plupart des programmes propose un travail à mener en équipes entrepreneuriales étudiantes. De plus, la volonté politique est clairement de proposer ces programmes à tous les étudiants quelle que soit leur formation. Ces démarches demandent un encadrement et un accompagnement importants et les ressources, en particulier humaines, ne sont aujourd'hui pas suffisantes pour le suivi de projets individuels. Dans ce qui est souvent le premier projet entrepreneurial de leur vie, ces jeunes sont des novices à tous les niveaux, en tant que personne, en tant qu'étudiant, en tant qu'entrepreneur ou même simplement membre d'une équipe entrepreneuriale (Günzel-Jensen et Robinson, 2017). Or, tous n'ont pas la même appétence à devenir entrepreneur. Il n'y a aucune raison a priori qu'ils perçoivent tous un projet entrepreneurial de la même manière et souhaitent y investir les mêmes efforts ou y prennent le même plaisir.

À cela s'ajoute le fait que, en dehors des formats très courts type hackathon davantage tournés vers la créativité que la création à proprement parler, les programmes entrepreneuriaux intégrant les étapes de faisabilité sont souvent relativement longs, dépassent régulièrement les 10 semaines et relèvent de l'apprentissage par projet. Nos travaux précédents montrent que cette durée s'accompagne de divergences de comportements au sein du projet entrepreneurial collectif. Cela est dû notamment à des degrés divers de motivation et d'investissement dans le projet, à des réactions émotionnelles positives ou négatives plus ou moins fortes face aux situations d'incertitude (Le Pontois et Jaillot, 2021) et à différents degrés de capacité de gestion du stress grâce aux stratégies de coping ou d'ajustement (Foliard et Le Pontois, 2017). Ces comportements sont orientés vers la protection du projet, de l'équipe ou vers un désengagement de l'étudiant vis-à-vis du projet (Le Pontois et Foliard, 2018).

Or, la démarche entrepreneuriale d'une équipe étudiante inclut de facto un processus d'essai erreur relativement différent des enseignements traditionnels plus prescriptifs. Ce processus peut déstabiliser l'étudiant et générer toutes sortes d'émotions qui vont soit le paralyser, soit au contraire le faire avancer, et ce de manière itérative. Pour un entrepreneur, l'échec est souvent vécu comme un événement émotionnellement fort (De Hoe et Janssen, 2014). À une moindre 
échelle et parce qu'ils sont novices dans tous les domaines, les étudiants vont vivre le projet et connaître des émotions fortes, positives ou négatives, impactant leur motivation et leur capacité à agir. Accompagner les étudiants à s'entreprendre suppose l'intégration de la gestion des émotions dans le dispositif pour leur permettre de capitaliser sur des émotions positives ou au contraire de lever des freins nés d'émotions négatives. Dans le même ordre d'idées, il nous semble important de mieux connaître la genèse de ces émotions mais nous ne disposons pas d'un cadre de référence ou de liens avérés entre des événements liés au projet entrepreneurial et les émotions perçues par les étudiants. De nature qualitative inductive, l'objectif de cette contribution est d'éclairer les liens entre des événements typiques vécus par les équipes, les émotions générées et les choix d'action de chaque étudiant afin d'adapter l'accompagnement pédagogique.

Les données analysées ici sont des récits réflexifs individuels. Écrits a posteriori par 66 étudiants engagés dans 13 équipes entrepreneuriales, ces récits ont pour but de conduire les étudiants à adopter une posture réflexive (métacognition) pour analyser leurs apprentissages. En reprenant l'histoire de ces projets, nous identifions cinq événements récurrents générant des émotions positives et négatives. Nous illustrons ensuite l'impact de la nature de ces émotions aux niveaux individuel et collectif sur les actions engagées et l'avancée du projet entrepreneurial. Nous proposons ensuite un retour à la littérature, en mobilisant en particulier les concepts de flow et de mémoire transactive, pour éclairer nos contributions.

\section{Cadre théorique et problématique}

Apprendre à s'entreprendre suppose une éducation en entrepreneuriat qui choisisse de valoriser l'être humain de manière holistique (dimensions cognitives, sociales, affectives et morales), la créativité et l'exploitation des opportunités dans un environnement complexe, l'autonomie dans les situations d'apprentissage, la prise de risque et le sens des responsabilités (Kyrö, 2015). Le dispositif pédagogique étudié est construit sur les quatre principes de l'éducation de l'esprit d'entreprendre proposés par Verzat (2011) : 1/ apprendre à partir de problèmes réels en expérimentant des projets d'innovation inscrits dans une pédagogie active, $2 /$ développer la prise de responsabilité des apprenants grâce à un accompagnement adapté, 3/ apprendre en équipe hors les murs, 4/ bénéficier de rétroactions sur l'action réalisée grâce à l'évaluation formative par des acteurs internes et externes à l'institution. Dans des cadres d'apprentissages ouverts où les résultats ne sont pas à atteindre mais à construire, les étudiants sont invités à 
adapter leur manière de penser. S'appuyant sur les principes de Verzat (2011), Bornard et Briest-Breda (2014) identifient trois points clefs pour développer l'agilité cognitive des étudiants : 1/ nécessité d'une problématique non formalisée (cadre normatif avec énoncé ouvert et méthode), 2/ développement de l'agilité cognitive individuelle par le collectif, 3/ créer « un espace-temps d'agilité » (alternance contenu/processus réflexif dans un dispositif lui-même agile, créé par des enseignants agiles).

La réussite ou plus exactement la capacité d'une équipe entrepreneuriale étudiante à réaliser son projet tient en grande partie au maintien d'un niveau de motivation individuelle et collective à œuvrer dans le même sens, alors même qu'elle est confrontée sans cesse à un environnement incertain. Quelles que soient les émotions, positives ou négatives, ressenties au cours de la conduite du projet, les capacités d'adaptation des individus face au stress au sein d'un collectif impactent les comportements et la motivation. Ces liens réciproques entre motivation et émotions sont largement étudiés par la littérature en psychologie.

La motivation est considérée comme une source d'énergie, une direction ou encore la persévérance que les individus éprouvent dans leurs intentions ainsi que dans leurs actions (Ryan et Deci, 2000). Selon la théorie de l'autodétermination de Deci (1975) et de Deci et Ryan (1985, 1991), trois besoins psychologiques sont à la base de la motivation humaine : le besoin d'autonomie, le besoin de compétence et le besoin d'appartenance sociale. Lorsque ces trois besoins sont comblés, un sentiment de bien-être devrait être ressenti. Cette motivation peut être intrinsèque (la satisfaction est ressentie par la production d'une activité) ou extrinsèque (l'activité est pratiquée pour des raisons externes à l'individu). La motivation peut aussi se définir comme la volonté de changement (Baumeister, 2016) ou plus exactement comme la volonté et la recherche de ce changement (Reeve, 2016). Ces processus personnels donnent l'énergie, dirigent et maintiennent certains comportements (Reeve, 2016). Cependant, cette motivation n'est pas stable et peut varier en fonction des besoins, des réflexions comme les objectifs, les attentes ou valeurs, mais surtout des émotions.

L'émotion, en tant qu'objet d'étude, n'a pas de définition conceptuelle délimitée ${ }^{1}$. L'approche multi-componentielle présente les émotions comme la résultante de trois composantes :

\footnotetext{
${ }^{1}$ Les émotions sont ici considérées selon un paradigme constructiviste qui postule que les émotions sont issues d'un consensus social et qu'en ce sens les concepts liés aux émotions ne doivent pas être réifiés, ni admis comme représentant un phénomène réel. Les émotions sont une réalité mais la manière de les classer et de les nommer est une construction artificielle issue de travaux académiques, d'un langage et d'un contexte social restreint.
} 
physiologique, subjective, expressive et comportementale (Tcherkassof et Frijda, 2014). Les émotions qui nous intéressent ici sont celles reliées au projet entrepreneurial. Elles apparaissent comme des réactions complexes mais coordonnées aux événements significatifs de la vie de l'étudiant durant son projet. Pour Izard (1993), les émotions sont des manifestations subjectives (ressentis), psychologiques (le sens du devoir, l'urgence perçue de s'adapter), expressives (verbalement et non verbalement) et des tendances à l'action (préparation physiologique à agir). Les émotions sont de brèves poussées de comportements adaptatifs qui dirigent l'attention vers l'élément déclencheur. Pekrun et Frese (1992) ont été les précurseurs dans l'étude des émotions les plus fréquemment ressenties et exprimées dans l'entreprise et soulignent deux dimensions importantes pour l'étude des équipes entrepreneuriales étudiantes : 1) la valence qui correspond au caractère plaisant ou non d'une tâche et qui amène des émotions positives quand la performance réelle est proche de la performance attendue et 2) le focus qui correspond aux émotions générées par une tâche ou par autrui (point nous intéressant particulièrement ici).

Dans le cas de ressentis d'émotions négatives, dont notamment l'appréhension ou la peur, une réaction adaptative de stress peut émerger. Les stratégies dites de coping (d'ajustement) désignent l'ensemble des comportements d'adaptation qu'un individu met en œuvre entre lui et un événement perçu comme menaçant (stresseur) pour maîtriser, tolérer ou diminuer l'impact de celui-ci sur son bien-être psychologique et physique (Paulhan, 1992). Selon Lazarus et Folkman (1984), le coping désigne "l'ensemble des efforts cognitifs et comportementaux destinés à maîtriser, réduire ou tolérer les exigences internes ou externes qui menacent ou dépassent les ressources d'un individu ». Ces stratégies peuvent être positives ou négatives (Côté, 2013) selon que l'étudiant pense pouvoir contrôler la situation, évitantes ou vigilantes si elles sont centrées sur l'émotion ou sur le problème (Ionescu, Jaquet et Lhote, 1997). Ces stratégies peuvent conduire à renforcer l'équipe et permettre à ses membres de développer leur apprentissage ou au contraire bloquer le fonctionnement collectif et conduire à des comportements de désengagement et de retrait du projet. Pour autant, dans les deux cas, le projet peut être protégé par l'équipe (obtenir des résultats académiques et opérationnels) ou au contraire désinvesti. 


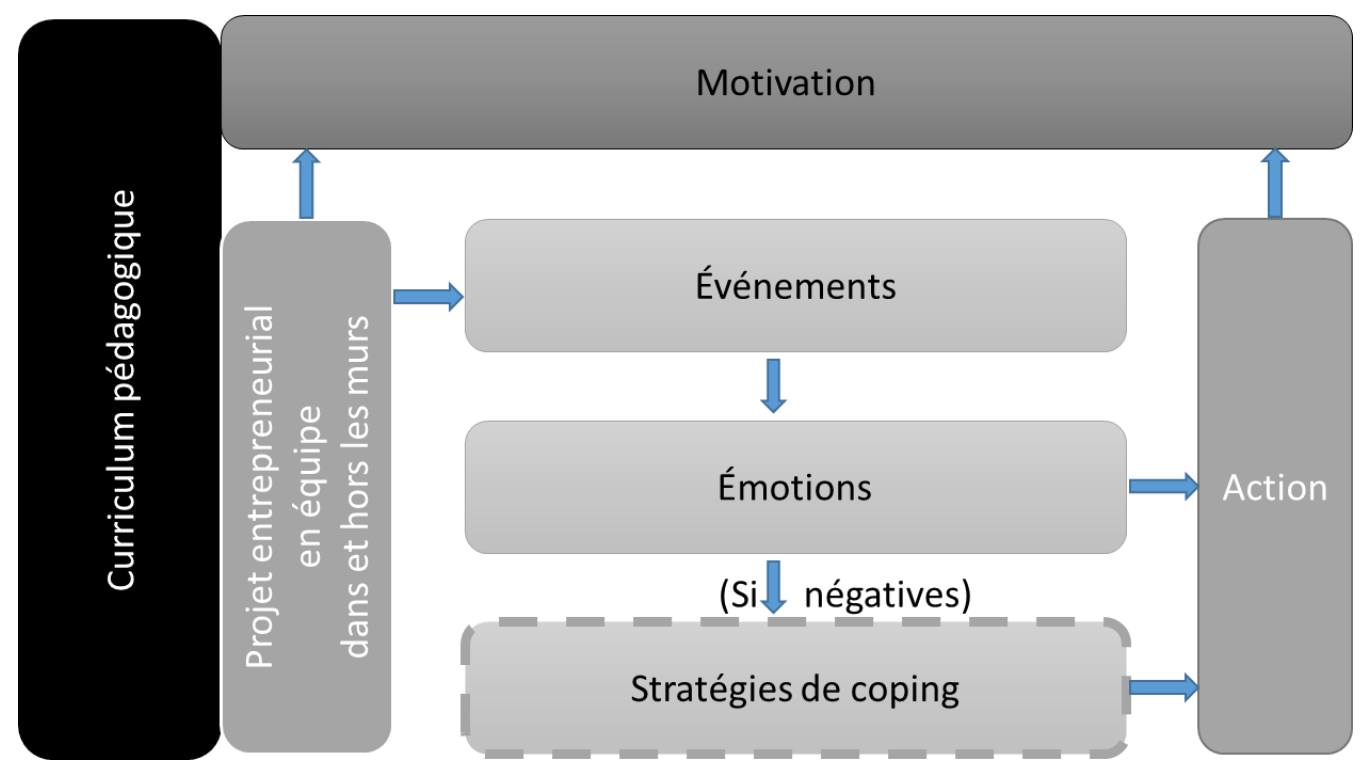

Figure 1 - Articulation du cadre conceptuel de la recherche

Ainsi, les émotions donnent de la valeur aux choses, aux tâches et aux travaux réalisés par les étudiants dans leur projet entrepreneurial. Elles ont un impact sur les motivations qui poussent à l'action ou au contraire qui génèrent des comportements de désengagement du projet, selon les stratégies de coping investies. Vouloir accompagner la motivation renvoie donc à la compréhension de la genèse, de l'élément déclencheur des émotions qui la sous-tendent. Pour ce faire, nous souhaitons dans cette proposition explorer quelles sont les émotions générées par un projet entrepreneurial, à quelles étapes du processus entrepreneurial et avec quelles conséquences sur la motivation et l'action. Identifier des réactions émotionnelles face à des événements typiques de projets entrepreneuriaux permet d'adapter les enseignements et l'accompagnement afin de répondre aux besoins des équipes étudiantes avant une trop grande perte de motivation. La figure 1 synthétise les articulations entre les différents concepts que nous mobilisons. Notre recherche se concentre ici sur les relations entre événements et émotions et plus particulièrement sur la nature des émotions générées, sur leur valence positive ou négative dont la littérature a montré l'impact sur la motivation. 


\section{Méthodologie}

Nous avons privilégié une approche qualitative basée sur des récits individuels d'expérience entrepreneuriale vécue par les étudiants. Notre échantillon est composé de 13 équipes de projet, soit 66 étudiants inscrits en deuxième cycle ${ }^{2}$ dans 2 écoles de commerce (5 équipes multiculturelles dans une école européenne, 8 équipes dans une école française). Dans les deux écoles, les étudiants volontaires pour cet électif ont réalisé un projet entrepreneurial en 3 mois (école européenne) et 5 mois (école française). Ils ont dû pour cela trouver une idée et réaliser les principales tâches liées à la faisabilité d'un projet entrepreneurial (étude de marché, montage financier, business model, etc.). Pour affiner leur projet, les étudiants sont allés collecter de l'information auprès d'utilisateurs potentiels et de professionnels du secteur. La tâche finale consiste en un concours de pitchs devant un jury d'investisseurs professionnels. À la fin de cette expérience, chaque étudiant devait faire un retour d'expérience à travers un récit écrit répondant à quatre thématiques : 1) Raconter les faits ; 2) Analyser les ressentis associés à ces faits ; 3) Tirer les leçons ; 4) Prévoir l'action future. Les récits individuels représentent $40 \%$ de la note du cours. Les $60 \%$ restants sont attribués collectivement (présentation orale + dossiers de business plan). Une grille critériée de notation du récit individuel est fournie aux étudiants en amont. Les points sont attribués selon que les critères sont non vérifiés ( 0 point $)$, partiellement vérifiés (1 point) ou totalement vérifiés ( 2 points). Les critères sont les suivants : faits précis, faits significatifs, clarification de l'action de chaque membre, ressentis repérés chronologiquement, ressentis analysés pour soi, sensibilité aux ressentis des autres membres, nombre de leçons tirées $>=3$, leçons des réussites comme des échecs, repérage des ressources les plus aidantes, nombre de résolutions prises >=3, lisibilité du récit, longueur >1000 mots. Les récits comportent en moyenne 1650 mots par étudiant (3 à 10 pages) et ont été collectés en 2018.

Pour analyser ces récits, les deux chercheurs se sont appuyés sur le logiciel NVivo qui permet de coder les verbatim. Le codage visait essentiellement à relier des émotions à des événements. Le codage des émotions est réalisé à partir des mots et expressions-clefs identifiés dans les écrits des étudiants, que ce soit par proxémie directe (synonymie) ou formes ou racines associées. Nous nous sommes servis des scénarios d'EMOTAIX pour identifier le lexique émotionnel et affectif (Piolat et Bannour, 2009), EMOTAIX proposant un classement des

\footnotetext{
${ }^{2}$ En Master 1 et Master 2 dans le système LMD français.
} 
émotions en catégories de plus de 2000 référents $^{3}$. Nous avons commencé l'analyse en identifiant les concepts initiaux dans les données et en les regroupant en catégories (codage ouvert). Pour effectuer ce codage initial des données, nous avons essayé de maintenir l'intégrité du codage conceptuel de premier ordre (Gioia et al., 2013) en utilisant des codes in vivo (Corbin et Strauss, 1990) ou de simples phrases de description (Corley et Gioia, 2004). Cette partie nous permet de commencer un recueil complet des termes de premier ordre, à qualifier la perception et le ressenti des étudiants, de quoi ils parlent et comment ils en parlent. Nous avons commencé à coder en parallèle les mêmes récits puis nous nous sommes accordés sur les termes. Les récits ont ensuite été répartis entre les deux chercheurs et seuls les nouveaux codes ont été discutés. Nous avons ensuite réalisé en commun un travail d'abstraction pour identifier de manière inductive les thèmes de second ordre que nous avons également catégorisés. Le codage secondaire a permis d'attribuer des sens communs et de regrouper les occurrences par différenciation et similitude. Le registre des émotions relevées reste moyen sans aller dans les excès positifs ou négatifs. Pour le codage final, nous avons repéré 7 catégories d'émotions positives selon EMOTAIX (sang-froid calme, bien-être bonheur, bien-être lucidité, bien-être entrain, bien-être soulagement, bien-être satisfaction et bienveillance) et 6 catégories d'émotions négatives (anxiété crainte, anxiété tension, mal-être dépression, mal-être trouble, mal-être frustration, malveillance agressivité) représentant la quasi-totalité des émotions recensées (tableau 1).

Parallèlement, les étudiants ont exprimé les événements vécus par leur équipe entrepreneuriale. Nous avons utilisé 20 codes pour ces événements que nous avons choisi d'agréger en 5 catégories sous les dénominations suivantes : 1) l'origine de l'équipe et sa formation, 2) la phase de créativité, 3) les travaux d'analyse, de faisabilité et de développement, 4) l'organisation et la réorganisation du travail en équipe, son fonctionnement et 5) l'avis des autres incluant la présentation finale.

À partir des valeurs de codage des 66 récits, nous avons cherché à identifier des relations entre les événements et les émotions.

\section{Tableau 1 - Catégories d'émotions recensées dans les écrits des étudiants, d'après le scénario EMOTAIX}

\footnotetext{
${ }^{3}$ EMOTAIX est un dictionnaire organisé de plus de 2000 référents (sens propre et sens figuré). Il permet d'identifier et de catégoriser le lexique de l'émotion contenu dans des textes produits par oral ou par écrit. Ce lexique concerne les états psychologiques suivants : émotions, sentiments, humeurs, affects, personnalité émotionnelle, tempéraments.
} 


\begin{tabular}{|l|l|}
\hline \multirow{5}{*}{ Émotions positives } & sang-froid calme \\
\cline { 2 - 2 } & bien-être bonheur \\
\cline { 2 - 3 } & bien-être lucidité \\
\cline { 2 - 3 } & bien-être entrain \\
\cline { 2 - 3 } & bien-être soulagement satisfaction \\
\hline & bienveillance \\
\hline \multirow{5}{*}{ Émotions négatives } & anxiété crainte \\
\cline { 2 - 3 } & anxiété tension \\
\cline { 2 - 3 } & mal-être dépression \\
\cline { 2 - 3 } & mal-être trouble \\
\hline & mal-être frustration \\
\cline { 2 - 3 } & malveillance agressivité \\
\hline
\end{tabular}

\section{Résultats, identification des émotions et des événements}

Faire travailler les étudiants en équipe sur des projets entrepreneuriaux est souvent une nécessité liée aux moyens humains disponibles pour encadrer de tels travaux ainsi que par la nature même du projet entrepreneurial qui nécessite de savoir être et agir avec les autres (collaboration et coopération-Verzat, 2011). Cette facilité ne doit cependant pas masquer les difficultés liées à l'exercice souvent nouveau du travail en équipe sur des projets où l'autonomie, la créativité ou le sens des responsabilités de chacun et de tous sont nécessaires pour atteindre un certain niveau de performance. Or cette coopération n'est pas acquise d'emblée (Verzat et O'Shea, 2019) et de nombreux ajustements sont réalisés dans les équipes pour réaliser la production attendue. Notre intuition de départ née de notre expérience était que la motivation et l'implication de chaque membre de l'équipe n'est pas une constante. Nous nous sommes penchés plus précisément sur les événements marquant la vie de l'équipe et les émotions que chacun de ces 
événements pouvait générer. Nous montrons que ces émotions sont particulièrement présentes et de nature à impacter le projet dans sa globalité.

\section{Émotions exprimées par les étudiants}

Nous avons d'abord souhaité identifier les émotions rencontrées dans chaque équipe en distinguant les émotions positives et négatives. Les émotions positives sont en moyenne deux fois plus nombreuses que les émotions négatives. Les données agrégées des 13 équipes (graphe 1) montrent que les émotions positives dominantes en utilisant la grille EMOTAIX sont l'entrain et la satisfaction. L'entrain recouvre également l'excitation et l'enthousiasme, la satisfaction incluant la fierté, le plaisir, l'épanouissement et l'accomplissement. Les émotions négatives sont majoritairement la frustration et la dépression. La frustration inclut le déplaisir, et le doute, la dépression comprend également la tristesse, la déception et l'ennui.

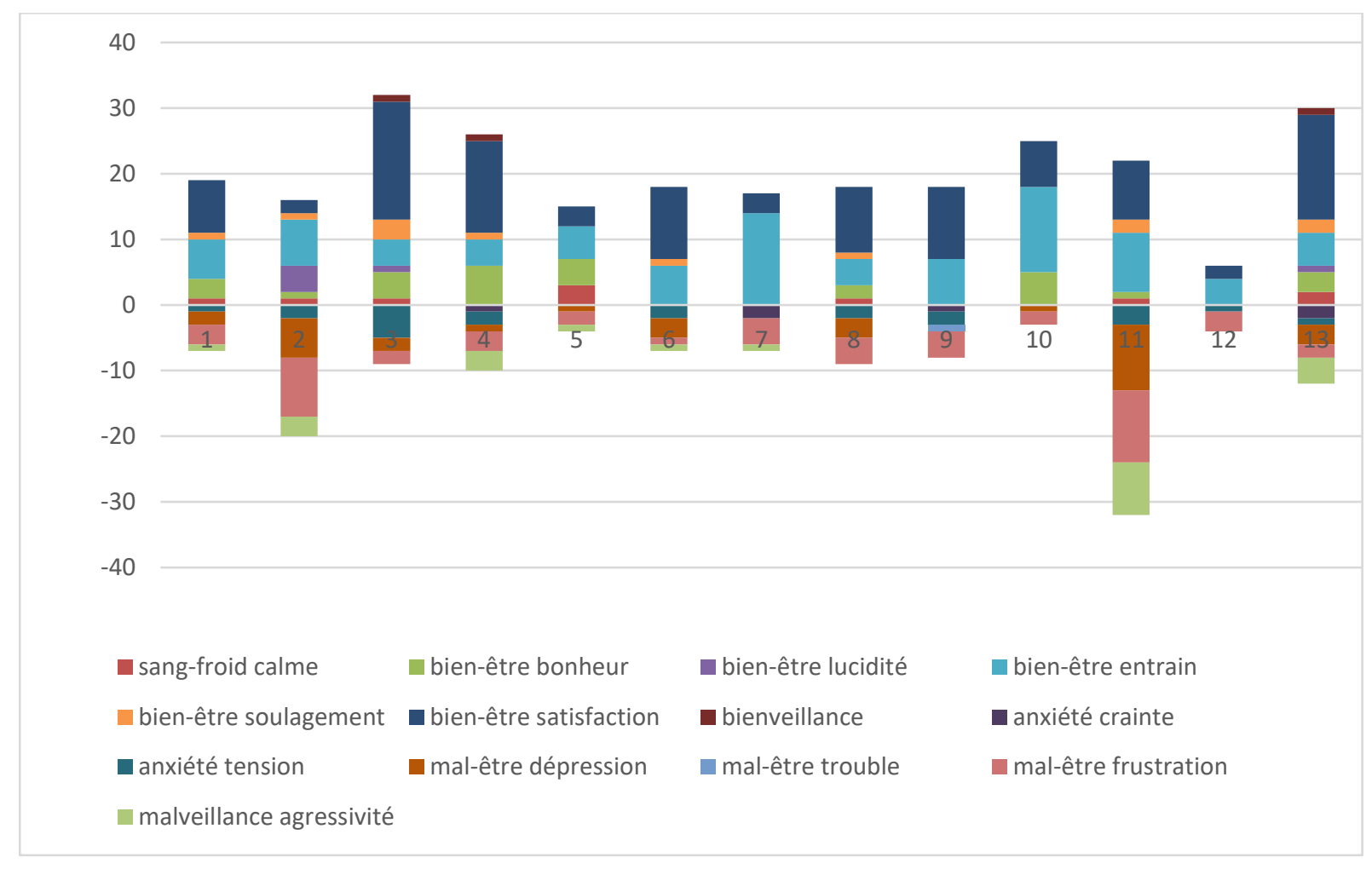

Graphe 1 - fréquences des émotions exprimées par équipe 
Tableau 2 - fréquences des émotions exprimées par équipe

\begin{tabular}{|c|c|c|c|c|c|c|c|c|c|c|c|c|c|c|}
\hline Équipe & 1 & 2 & 3 & 4 & 5 & 6 & 7 & 8 & 9 & 1 & 11 & 12 & 13 & Total \\
\hline sang-froid calme & 1 & 1 & 1 & & 3 & & & 1 & & & 1 & & 2 & 10 \\
\hline bien-être bonheur & 3 & 1 & 4 & 6 & 4 & & & 2 & & 5 & 1 & & 3 & 29 \\
\hline bien-être lucidité & & 4 & 1 & & & & & & & & & & 1 & 6 \\
\hline bien-être entrain & 6 & 7 & 4 & 4 & 5 & 6 & 14 & 4 & 7 & 13 & 9 & 4 & 5 & 88 \\
\hline bien-être soulagement & 1 & 1 & 3 & 1 & & 1 & & 1 & & & 2 & & 2 & 12 \\
\hline bien-être satisfaction & 8 & 2 & 18 & 14 & 3 & 11 & 3 & 1 & 11 & 7 & 9 & 2 & 16 & 105 \\
\hline bienveillance & & & 1 & 1 & & & & & & & & & 1 & 3 \\
\hline Total émotion > & 19 & 16 & 32 & 26 & 15 & 18 & 17 & 9 & 18 & 25 & 22 & 6 & 30 & 253 \\
\hline anxiété crainte & & & & 1 & & & 2 & & 1 & & & & 2 & 6 \\
\hline anxiété tension & 1 & 2 & 5 & 2 & & 2 & & 2 & 2 & & 3 & 1 & 1 & 21 \\
\hline mal-être dépression & 2 & 6 & 2 & 1 & 1 & 3 & & 3 & & 1 & 1 & & 3 & 23 \\
\hline mal-être trouble & & & & & & & & & 1 & & & & & 1 \\
\hline mal-être frustration & 3 & 9 & 2 & 3 & 2 & 1 & 4 & 4 & 4 & 2 & 11 & 3 & 2 & 50 \\
\hline malveillance agressivité & 1 & 3 & & 3 & 1 & 1 & 1 & & & & 8 & & 4 & 22 \\
\hline total émotion < & 7 & 20 & 9 & 10 & 4 & 7 & 7 & 9 & 8 & 3 & 23 & 4 & 12 & 123 \\
\hline émotion $>-<$ & 12 & -4 & 23 & 16 & 11 & 11 & 10 & 0 & 10 & 22 & -1 & 2 & 18 & \\
\hline
\end{tabular}

Sans aucune valeur statistique, nous avons comparé la fréquence des émotions positives auxquelles nous avons soustrait les émotions négatives (tableau 2). Deux équipes sur treize ressortent avec plus de négatifs que de positifs. Ce sont aussi celles qui ont connu des résultats opérationnels et académiques inférieurs aux autres (travail moins approfondi et notes plus basses).

\section{Événements à l'origine des émotions exprimées}

La vie de chaque équipe et de ses membres présente de grandes disparités que nous souhaitons éclairer en identifiant les événements à l'origine de ces émotions. Le graphe 2 reprend de manière synthétique les catégories d'événements agrégées de chaque projet avec les fréquences de verbatim. Le tableau 3 reprend les fréquences des verbatim par catégorie d'événements et par équipe. Comme ces projets sont d'une durée conséquente, les événements les plus fréquemment cités sont le déroulement du projet et le travail en équipe incluant la répartition des tâches. 


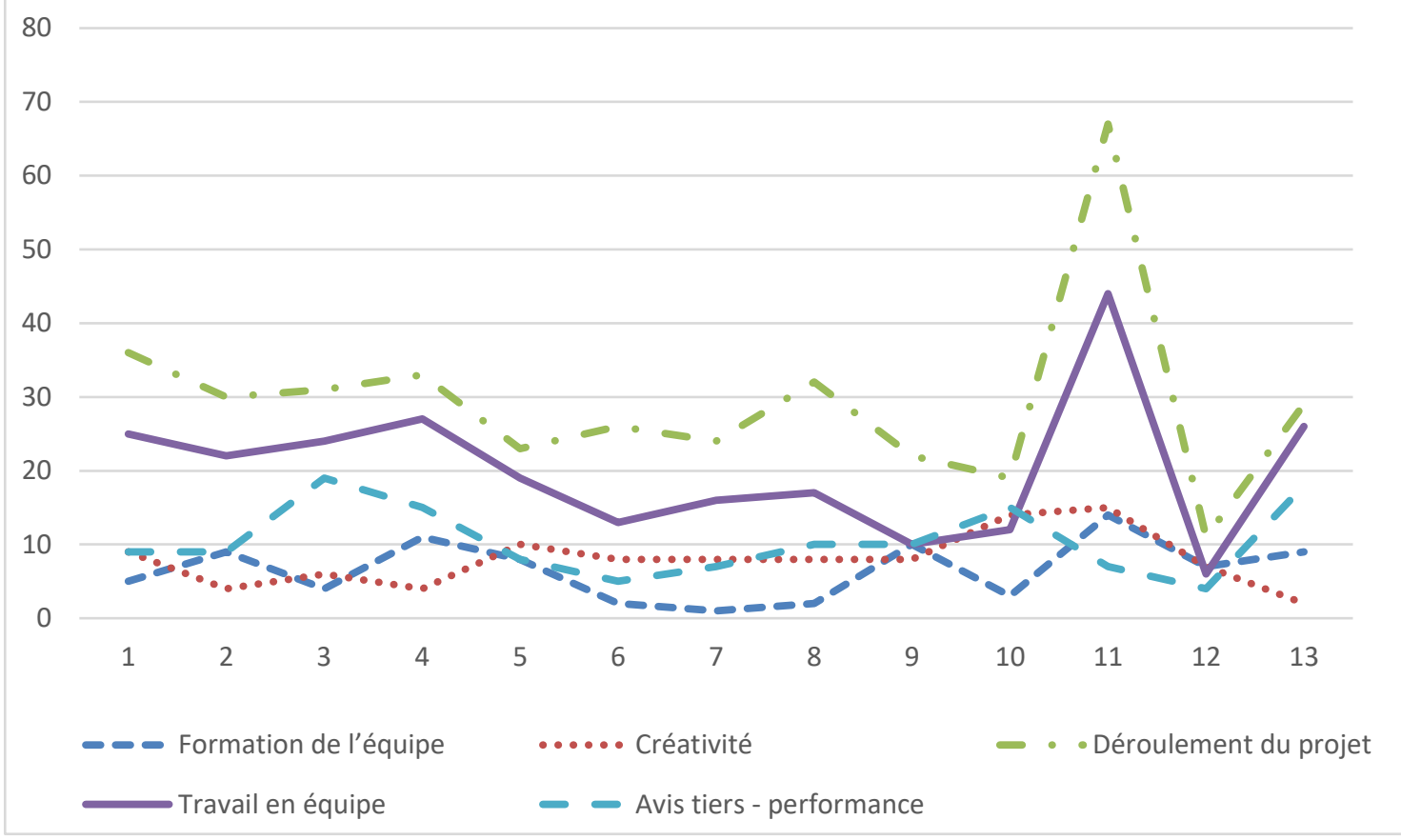

Graphe 2 - fréquence des verbatim par catégorie d'événements et par équipe.

Tableau 3 - fréquence des verbatim par catégorie d'événements et par équipe.

\begin{tabular}{|c|c|c|c|c|c|c|c|c|c|c|c|c|c|c|}
\hline $\begin{aligned} \text { Équipes } \\
\text { Événements }\end{aligned}$ & 1 & 2 & 3 & 4 & 5 & 6 & 7 & 8 & 9 & 10 & 11 & 12 & 13 & Total \\
\hline $\begin{array}{l}\text { formation de } \\
\text { l'équipe }\end{array}$ & 5 & 9 & 4 & 11 & 8 & 2 & 1 & 2 & 10 & 3 & 14 & 7 & 9 & 85 \\
\hline créativité & 9 & 4 & 6 & 4 & 10 & 8 & 8 & 8 & 8 & 14 & 15 & 7 & 2 & 103 \\
\hline $\begin{array}{l}\text { déroulement du } \\
\text { projet }\end{array}$ & 36 & 30 & 31 & 33 & 23 & 26 & 24 & 32 & 22 & 19 & 67 & 11 & 29 & 383 \\
\hline $\begin{array}{l}\text { répartition des } \\
\text { tâches }\end{array}$ & 25 & 22 & 24 & 27 & 19 & 13 & 16 & 17 & 10 & 12 & 44 & 6 & 26 & 261 \\
\hline $\begin{array}{l}\text { avis tiers - } \\
\text { performance }\end{array}$ & 9 & 9 & 19 & 15 & 8 & 5 & 7 & 10 & 10 & 15 & 7 & 4 & 18 & 136 \\
\hline total & 84 & 74 & 84 & 90 & 68 & 54 & 56 & 69 & 60 & 63 & 147 & 35 & 84 & \\
\hline
\end{tabular}

\section{Événements et conséquences émotionnelles}

Si ces informations «génériques » sont importantes, caractériser les émotions rencontrées par nos 66 étudiants et les événements qui impactent leur équipe puis croiser les deux peut nous 
donner un éclairage plus fin sur le fonctionnement des équipes et l'accompagnement que nous pouvons leur proposer. Les tableaux suivants présentent un ensemble de verbatim reprenant selon leur valence positive ou négative les émotions principales exprimées par les étudiants pour chacun des cinq événements identifiés par l'ensemble des équipes.

La formation de l'équipe (tableau 4) et la phase ${ }^{4}$ de créativité (tableau 5) sont des phases de découverte, de curiosité dans lesquelles les émotions positives dominent. Cependant, pour deux des équipes observées ( 2 et 11), la formation du groupe a été vécue comme difficile et contrainte avec des a priori négatifs dès la phase d'idéation qui se sont renforcés au fil du temps. Une seule équipe (5) s'est formée sur la volonté de faire des choses ensemble et a réussi à surmonter de nombreuses difficultés.

\section{Tableau 4 - Verbatim des émotions générées par l'événement « Formation de l'équipe »}

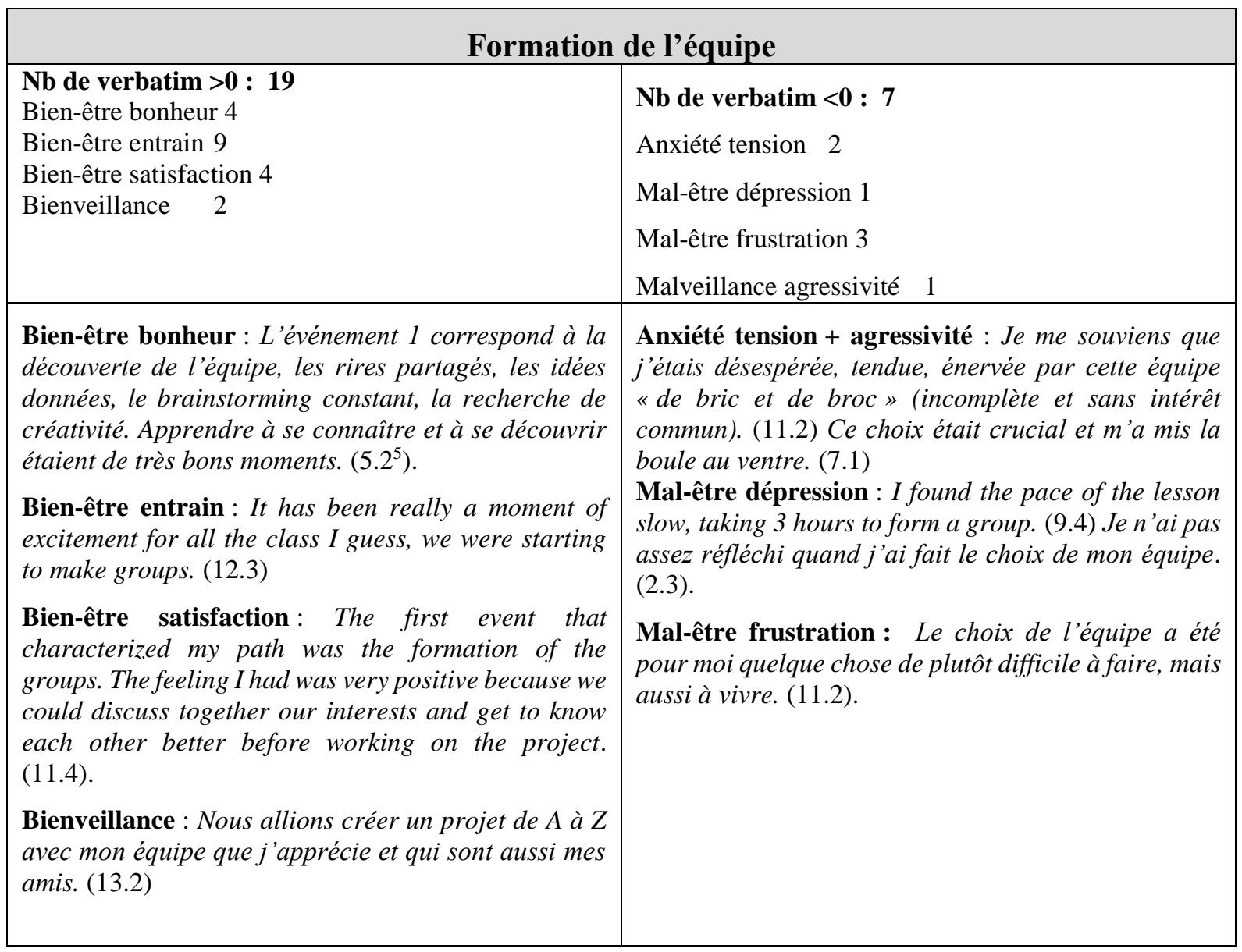

\footnotetext{
${ }^{4}$ Les phases se distinguent des événements. Elles correspondent à des périodes de temps consacrées à une étape du processus entrepreneurial. Pendant cette période, des événements se produisent et génèrent les émotions qui nous intéressent. La phase de créativité par exemple peut inclure à un moment un brainstorming lui-même générateur de frustration ou d'enthousiasme.

${ }^{5}$ Les verbatim sont présentés avec le numéro de l'équipe en premier, de 1 à 13 puis le numéro de l'étudiant(e).
} 
Tableau 5 - Verbatim des émotions générées par l'événement « Créativité »

\begin{tabular}{|c|c|}
\hline \multicolumn{2}{|c|}{ Créativité } \\
\hline $\begin{array}{l}\text { Nb de verbatim >0 : } 40 \\
\text { Sang-froid calme } 2 \\
\text { Bien-être bonheur } 8 \\
\text { Bien-être entrain } 23 \\
\text { Bien-être satisfaction } 7\end{array}$ & $\begin{array}{l}\text { Nb de verbatim <0 : } 12 \\
\text { Anxiété tension } 2 \\
\text { Mal-être dépression } 1 \\
\text { Mal-être frustration } 8 \\
\text { Malveillance agressivité } 1\end{array}$ \\
\hline $\begin{array}{l}\text { Sang-froid calme : Suite à la validation de l'idée, } \\
\text { nous sommes tous pleins de joie et optimistes } \\
\text { concernant notre projet car c'est un secteur très } \\
\text { porteur. De plus, suite à un brainstorming les idées ne } \\
\text { manquent pas, on ne voit presque plus le blanc du } \\
\text { tableau. (1.5). } \\
\text { Bien-être bonheur: Plusieurs moments de cette } \\
\text { première partie de la construction du projet ont été } \\
\text { source de joie et d'amusement. (3.3). } \\
\text { Bien-être entrain : Le projet a émergé très vite il a } \\
\text { suscitél'enthousiasme du groupe et nous avons décidé } \\
\text { de nous lancer avec notre fraîcheur et notre } \\
\text { enthousiasme dedans. (10.1). } \\
\text { Bien-être satisfaction : Nous étions fiers et sûrs de } \\
\text { nous (10.2). Le brainstorming est mon moment préféré } \\
\text { lors des étapes de réalisation d'un projet. J'apprécie } \\
\text { particulièrement ce moment où les idées fusent et où il } \\
\text { n'y a aucune censure pour tirer un maximum de } \\
\text { choses de ces séances. (1.2) }\end{array}$ & $\begin{array}{l}\text { Anxiété tension : Étant donné que je suis quelqu'un } \\
\text { qui essaye de prendre le moins de risque possible, } \\
\text { cette idée de repartir à } 0 \text { m'inquiétait : si au bout de } \\
\text { la } 3-4^{e} \text { séance nous repartions à } 0 \text {, qu'est-ce qui me } \\
\text { prouve que nous ne ferions pas de même avec la } \\
\text { deuxième idée? (6.3) } \\
\text { Mal-être dépression : La première séance une fois } \\
\text { l'équipe réunie était une véritable douchefroide. Nous } \\
\text { avions réuni l'équipe mais on était incapable de se } \\
\text { mettre d'accord sur une idée. Nous avions } \\
\text { brainstormé mais les idées ne plaisaient pas à tout le } \\
\text { monde. (5.3) } \\
\text { Mal-être frustration : Il a fallu beaucoup de } \\
\text { négociations et de conviction dans nos propos pour } \\
\text { qu'il arrête de dire noir dès que quelqu'un disait } \\
\text { blanc. (5.2). } \\
\text { Malveillance agressivité : Cette idée ne me plaisait } \\
\text { pas du tout. [...] Sentiment ressenti: énervement. (6.2) }\end{array}$ \\
\hline
\end{tabular}

Le déroulement du projet (tableau 6) et le travail en équipe (tableau 7) correspondent à la phase de recherche d'information et de production dans des délais contraints. Cette phase est la plus longue dans le processus et génère un nombre nettement plus important d'expressions des événements vécus (67\% du total). C'est une phase plus difficile de collecte et d'analyse de données, de concrétisation des idées et de décisions à prendre. La concrétisation génère pour l'ensemble des équipes des émotions positives. Les émotions négatives sont majoritairement dues à une gestion de projet difficile, la charge de travail et la qualité de la production. Le travail en équipe (tableau 7) est la phase qui génère le plus d'émotions, 110 au total, et concentre plus de $40 \%$ des émotions négatives. Ces émotions sont générées par des différences dans la charge de travail et les différences d'implication perçues des membres de l'équipe empêchant une collaboration efficace. 
Tableau 6 - Verbatim des émotions générées par l'événement « Déroulement du projet »

\begin{tabular}{|c|c|}
\hline \multicolumn{2}{|c|}{ Déroulement du projet } \\
\hline $\begin{array}{l}\text { Nb de verbatim }>\mathbf{0}: \mathbf{4 5} \\
\text { Sang-froid calme }: 2 \\
\text { Bien-être bonheur : } 6 \\
\text { Bien-être entrain }: 17 \\
\text { Bien-être soulagement : } 4 \\
\text { Bien-être satisfaction }: 16\end{array}$ & $\begin{array}{l}\text { Nb de verbatim }<\mathbf{0}: \mathbf{2 5} \\
\text { Anxiété crainte : } 2 \\
\text { Anxiété tension : } 6 \\
\text { Mal-être dépression : } 5 \\
\text { Mal-être frustration : } 11 \\
\text { Malveillance agressivité : } 1\end{array}$ \\
\hline $\begin{array}{l}\text { Sang-froid calme : Les dernières étapes se sont } \\
\text { ensuite déroulées de façon plus sereine car nous étions } \\
\text { sûrs de nos idées et nous savions tous quel était } \\
\text { l'objectiffinal. (8.4) } \\
\text { Bien-être bonheur : Au plus nous avancions, au plus } \\
\text { j'étais heureuse. La portée réaliste du projet m'a en } \\
\text { effet boostée. Les différentes matières que nous } \\
\text { étudions, mêlées à notre travail nous ont permis de } \\
\text { rendre un projet à la base abstrait, concret et positif. } \\
\text { (10.2) } \\
\text { Bien-être entrain : Lorsque nous nous sommes remis } \\
\text { au travail, la confiance envers le projet est remontée } \\
\text { et nous avons réellement avancé. (8.5) } \\
\text { Bien-être soulagement : Personnellement, j'étais } \\
\text { soulagée que ce soit la fin. (4.4) } \\
\text { Bien-être satisfaction : Au final, les résultats ont été } \\
\text { très positifs et nous avons avancé en respectant } \\
\text { chacune des deadlines imposées. (8.1) }\end{array}$ & $\begin{array}{l}\text { Anxiété crainte : Pendant presque un mois nous ne } \\
\text { travaillons pas assez sur le projet, une heure par-ci } \\
\text { une heure par-là, mais nous avons l'impression d'être } \\
\text { au point mort. À ce moment-là, un peu d'anxiété se fait } \\
\text { ressentir. (1.5) } \\
\text { Anxiété tension : Nous n'avons pas pu faire le travail } \\
\text { de la qualité que nous souhaitions. Nous avons alors } \\
\text { peut-être eu un problème avec la pression de la } \\
\text { soutenance mais je pense réellement que pour avoir } \\
\text { un projet vraiment solide il nous aurait fallu plus de } \\
\text { temps. (8.2) } \\
\text { Mal-être dépression : À un moment précis, pendant } \\
\text { un de vos cours, je me souviens avoir été déçu par la } \\
\text { vision de mes camarades qui voyaient ce projet comme } \\
\text { un simple projet de cours destiné à avoir une note et } \\
\text { rien de plus, que ce ne sera pas concret. (8.3) } \\
\text { Mal-être frustration : Au cours du projet nous avons } \\
\text { parfois dî nous remettre en question et ce sentiment } \\
\text { n'était pas très agréable car cela nous stopper dans } \\
\text { notre avancement ce qui est très frustrant. (8.4) }\end{array}$ \\
\hline
\end{tabular}

Tableau 7 - Verbatim des émotions générées par l'événement « Travail en équipe »

\begin{tabular}{|c|c|}
\hline \multicolumn{2}{|c|}{ Travail en équipe } \\
\hline $\begin{array}{l}\text { Nb de verbatim : } 59 \\
\text { Sang-froid calme : } 2 \\
\text { Bien-être bonheur : } 3 \\
\text { Bien-être lucidité : } 3 \\
\text { Bien-être entrain : } 14 \\
\text { Bien-être soulagement : } 5 \\
\text { Bien-être satisfaction : } 32\end{array}$ & $\begin{array}{l}\text { Nb de verbatim : } \mathbf{5 1} \\
\text { Anxiété tension : } 7 \\
\text { Mal-être dépression : } 9 \\
\text { Mal-être trouble : } 1 \\
\text { Mal-être frustration : } 18 \\
\text { Malveillance agressivité : } 16\end{array}$ \\
\hline $\begin{array}{l}\text { Sang-froid calme : Elle a fait un très gros travail sur } \\
\text { la recherche concurrentielle et cela nous a permis de } \\
\text { nous conforter sur notre positionnement. (13.1) } \\
\text { Bien-être bonheur : Et je me rappelle de notre joie à } \\
\text { tous lorsque nous avons acté pour 10. Nous étions } \\
\text { fiers et sûrs de nous (10.2). }\end{array}$ & $\begin{array}{l}\text { Anxiété tension : Je suis épuisée, pendant } 1 \text { mois je } \\
n \text { 'avais plus envie de venir à l'école, je comptais sur } \\
\text { la bonne humeur et les énergies positives que j'avais } \\
\text { dans mon environnement au travail (mon alternance) } \\
\text { et je redoutais de venir en classe. Je n'avais plus envie } \\
\text { de bosser pour } 5 .(2.3) \text {. }\end{array}$ \\
\hline
\end{tabular}


Bien-être lucidité : J'ai pris du recul et j'ai fait la part des choses, le tableau est ni tout noir ni tout blanc, il $y$ avait effectivement des choses pour lesquelles je devais me remettre en question. (2.2)

Bien-être entrain : Ce qui m'intéressait particulièrement était le fait d'innover ensemble, travailler en équipe pour un projet commun, commencer de rien et voir jusqu'où cela allait nous mener. (2.2)

Bien-être soulagement : Notre équipe soudée m'a permis de la dépasser. (3.2)

Bien-être satisfaction : Nous avons alors affiné par la suite le projet, tous les membres de l'équipe ont été pro actifs et ils aimaient travailler sur le projet je pense. Il y avait beaucoup d'échange de communication et nous fournissions un travail de qualité. (8.2)
Mal-être dépression : J'ai été déçu pendant ce projet car moi je considère que par amitié ils auraient pu $m$ 'aider beaucoup plus. (2.3)

Mal-être trouble : J'avais l'impression que chacun avait fait sa part et ceci très sérieusement pour tout le monde, mais que, pendant la présentation nous sommes restés chacun cloisonnés dans nos parties, ce qui est vraiment dommage. (9.5)

Mal-être frustration : Je ne pense pas que les membres de mon équipe ont connaissance du temps que j'ai pu passer à faire ce suivi personnalisé et j'ai pu me prendre quelques reproches concernant mes propres missions en retard. (2.4)

Malveillance agressivité : J'ai donc réuni les membres de mon équipe et j'ai exprimé ce que je ressentais la colère. (13.3)

Enfin, l'avis des autres sur la performance (tableau 8) est l'élément générant le plus d'émotions positives (71 positives pour 20 négatives). Bénéficier de l'avis de tiers faisant souvent autorité par leur légitimité ou leur expertise dans l'avancée du projet semble permettre à l'équipe de déminer des problèmes internes de prise de décision et conforter ou réconforter les membres dans leur travail. Généraliser l'intervention des tiers à des moments clés de l'avancée du projet peut être de nature à limiter les tensions et les émotions négatives dans les équipes : validation de l'idée d'affaire, validation d'un plan d'affaires, avancée à mi-parcours, etc.

Chaque projet a connu des tensions (32 sources pour 80 références) se manifestant par de la colère (11 références), de la frustration (10 références) et conduisent fréquemment à une baisse de motivation ( 9 références directes). Cette dernière est également générée par les difficultés liées à 1 'avancée du projet ( 8 références) et à l'avis des autres ( 7 références).

Les équipes les plus performantes ont « contrôlé » leur activité avec une bonne répartition des tâches, un respect des délais et des productions intermédiaires etc. et ont accordé de la valeur à sa réalisation, soit pour gagner le concours, soit pour obtenir une bonne note, soit pour obtenir des retours professionnels positifs, les trois raisons ne s'excluant pas les unes les autres. Les équipes ayant sous-performé (équipes 2 et 11), c'est-à-dire n'ayant pas atteint ces mêmes objectifs professionnels et académiques, ont perdu le contrôle des activités par manque de cohésion dans l'équipe, des retards dans le travail fait, ... Pour ces dernières, la valeur accordée au projet était la même que pour les autres équipes, mais elles ont généré plus d'émotions négatives (graphe 2). 
Tableau 8 - Verbatim des émotions générées par l’événement « Performance perçue par les autres »

\section{Performance perçue par les autres (dont jury final)}

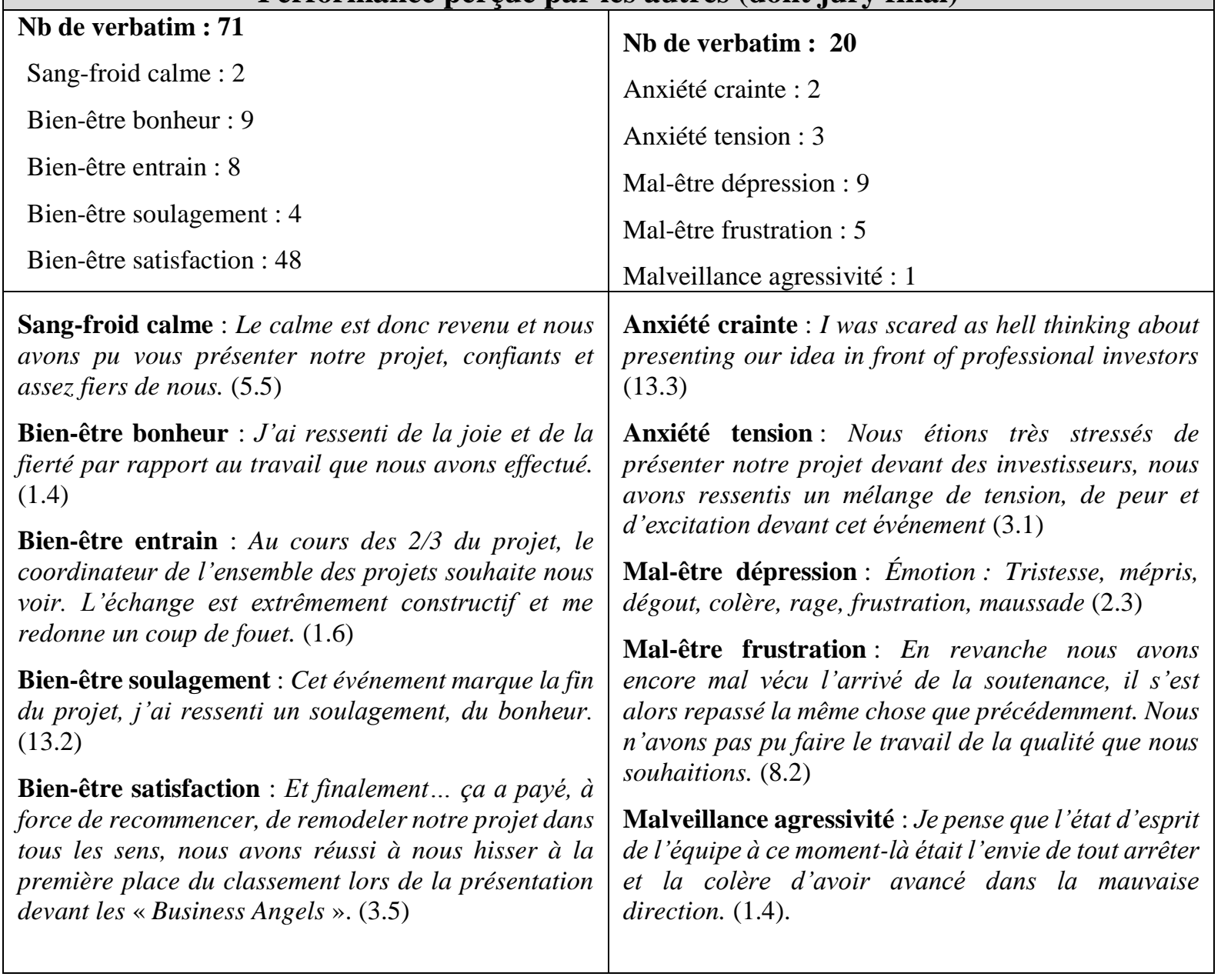

\section{Discussion}

Si la grande majorité des dispositifs d'éducation à l'entrepreneuriat adopte un format d'apprentissage expérientiel en équipe, c'est à la fois pour répondre à une volonté de coller au plus près à l'écosystème d'affaire réel et pour surmonter l'impossibilité d'un accompagnement individuel dans un contexte de massification des enseignements dans le supérieur. Pour autant, travailler en équipe n'est pas toujours inné, c'est une compétence qui s'apprend, s'entraine et s'accompagne (Verzat, 2011). Les événements vécus au cours d'un projet entrepreneurial génèrent de nombreuses émotions et actions en réaction. L'objectif de cette étude est d'éclairer les liens entre des événements typiques vécus par les équipes, les émotions générées et les choix d'action individuelle de chaque étudiant afin d'adapter l'accompagnement pédagogique. Notre recherche apporte deux contributions. 
D'une part, nous nommons et catégorisons selon leur valence (se sentir bien ou mal) les émotions ressenties par les étudiants au cours de l'expérience entrepreneuriale et exprimées en fin de processus. Nous identifions ensuite les événements typiques auxquels sont associées les émotions exprimées : la formation de l'équipe, la phase de créativité, le déroulement du projet, le travail en équipe et la performance perçue par les autres. Pour la majorité des équipes, la phase de découverte de constitution des équipes et de créativité s'accompagne d'émotions positives fortes comme l'excitation et l'enthousiasme, qui conduisent à un renforcement de la motivation. Les pics positifs apparaissent de fait lors de la constitution des équipes et de la phase d'idéation, puis les phases opérationnelles du projet. Ce sont les événements longitudinaux, déroulement du projet et travail en équipe, qui suscitent le plus d'émotions (positives et négatives) et cristallisent les baisses et hausses de motivation liées aux résultats des actions entreprises. Les stratégies de coping mises en œuvre par les équipes qui sont efficaces semblent centrées sur la protection du projet et/ou de l'équipe, avec une vision assez claire du degré de contrôlabilité de la situation et une capacité à capitaliser sur les émotions positives. Lorsque les émotions négatives dominent, ces équipes adoptent une stratégie de coping vigilante, orientée sur la résolution de problème. Les équipes en difficulté adoptent des stratégies évitantes centrées sur les émotions (négatives) avec un sentiment individuel de contrôlabilité de la situation faible.

D'autre part, nous montrons que les événements issus de ces projets génèrent en moyenne deux fois plus d'émotions positives que d'émotions négatives. De manière évidente du fait de sa durée, la phase opérationnelle de la conduite du projet entrepreneurial est celle qui génère le plus d'émotions, qu'elles soient positives ou négatives. Si les émotions positives exprimées $a$ posteriori sont deux fois plus nombreuses que les émotions négatives, la valence positive semble pourtant insuffisante à maintenir une motivation sur toute la durée du projet. Les équipes qui ne génèrent pas d'émotions positives lors de l'une des deux premières phases se trouvent en difficulté pour agir et perdent de la motivation.

Inscrite dans le cadre d'un enseignement de l'entrepreneuriat, cette étude fait écho par abduction à la théorie de Pekrun (2006) qui rend compte des antécédents et des effets des émotions en contexte d'apprentissage. Cette théorie est centrée sur les émotions d'accomplissement et le processus entrepreneurial est une suite d'actions susceptibles de déclencher ces émotions. Scherer (2001) les définit comme des réactions brèves à des événements avec des dimensions cognitive, physiologique, motivationnelle, expressive et affective que l'on retrouve de manière explicite dans les verbatim de nos étudiants 
entrepreneurs. Nous retrouvons également dans notre étude les émotions positives et négatives ressenties par les étudiants qui influencent selon Pekrun (2006) en plus ou en moins l'activation cognitive. Nous retrouvons dans nos résultats le lien entre l'émotion, la réaction instantanée (ou l'absence de réaction) et la projection dans l'action future. Nous repérons dans les verbatim du tableau 4 les deux critères subjectifs de la théorie de Pekrun que sont le contrôle et la valeur.

Inscrits dans un démarche abductive, nous avons opéré un retour à la littérature pour mettre en perspective nos résultats. Les concepts de flow (Csikszentmihalyi, 2002) et de mémoire transactive (Wegner, 1995) semblent pouvoir éclairer nos résultats. Ancré dans le champ de la psychologie positive, le flow est une expérience optimale positive caractérisée par un ancrage total de l'être dans le moment présent, alors même que le corps ou l'esprit de l'individu est confronté à ses limites dans un effort volontaire pour accomplir quelque chose de difficile. Le flow est un état de joie, de créativité et d'engagement total dans lequel les problèmes semblent disparaître, remplacés par un ressenti d'excitation, de contrôle et de transcendance. La phase de constitution de l'équipe et de créativité a généré des émotions qui ont nourri ce flow dans l'ensemble des équipes, hormis deux, qui ont fluctué entre deux extrêmes, l'anxiété (et ses corollaires, colère et stress) et l'apathie lorsque les choses ne font plus sens. La majorité des équipes semble avoir su capitaliser sur ce flow initial et le renforcer lors des phases de production. La mémoire transactive est la capacité des individus à partager des modèles mentaux communs pour créer des équipes efficaces (Wegner, 1995). Elle relève de la métacognition collective, c'est-à-dire d'une connaissance partagée sur la compétence respective de chacun des membres. Trois facteurs soutiennent le développement et la rétention d'un bon niveau de mémoire transactive (Woolley et al., 2015) : le type de tâche, la taille de l'équipe et le capital psychologique - par exemple l'assertivité, la capacité à s'exprimer et à défendre ses droits sans empiéter sur ceux des autres. Enfin, la mémoire transactive est liée au rendement des équipes étudiantes (coopération sur des projets communs ; Michinov et Michinov, 2009). Les deux équipes qui se sont considérées en échec ont éprouvé des difficultés de communication et n'ont pas réussi à générer le « commun » dans le projet. Elles sont restées bloquées au stade de groupe et n'ont pu devenir une équipe qui construit et développe une forme de mémoire transactive. Les émotions exprimées en début de projet entrepreneurial (formation d'équipe et créativité) semblent fortement prédictives de la capacité des groupes à se constituer en équipe efficace -c'est-à-dire une équipe qui développe sa mémoire transactive et capitalise sur les flows générés pour produire les livrables et des résultats d'apprentissage - et impactent fortement les actions et degré de motivation de la phase de production. 
Notre étude a également une contribution managériale. L'enjeu pour les accompagnateurs est d'identifier au plus tôt les équipes dysfonctionnelles grâce à un espace permettant l'expression des émotions liées aux deux premières phases du projet. Un accompagnement au travail en équipe est indispensable en amont de la phase de production pour éviter des situations de blocage et des moments souvent difficiles à vivre pour nos étudiants. Cela nous appelle à proposer des outils d'accompagnement opérant dès la phase de constitution des équipes et à poursuivre les travaux de Verzat et O’Shea (2019) ou de Paul (2009). Une réflexion précoce sur le fonctionnement d'équipe (Le Pontois et Foliard, 2018) centré sur l'expression des émotions semble pertinente à mettre en place dès le début du projet - et non pas en fin de projet uniquement via l'essai réflexif. Une piste serait la tenue d'un carnet de bord comportant une rubrique «événements et émotions positives ». Concernant l'impact de l'avis des autres sur l'efficacité de l'équipe, de nombreux formats sont à imaginer comme des commissions de validation intermédiaire, du peer coaching, etc.

\section{Conclusion}

Pour conclure, nous montrons que dans des projets entrepreneuriaux de longue durée, toutes les équipes connaissent des pics d'émotions positives sur lequel il semble intéressant de capitaliser et de maintenir le flow positif en travaillant la cohésion d'équipe et le niveau de curiosité nécessaire à ces émotions positives. Nous indiquons également que la principale difficulté générant la majorité des émotions négatives provient du travail en équipe et que nous ne pouvons plus partir du principe que les étudiants vont s'autogérer et développer d'eux-mêmes les compétences sociales nécessaires. Un recours à la psychologie positive pour rééquilibrer les émotions ainsi que des outils pour développer la mémoire transactive semblent souhaitables.

Notre travail souligne enfin la grande hétérogénéité de fonctionnements et de trajectoires émotionnelles des équipes observées. Une limite de notre étude est le faible nombre d'équipes qui ne permet en aucun cas une généralisation des résultats. Étendre le dispositif d'observation à un nombre plus important d'équipes est un travail en cours. L'objectif est de réaliser une étude quantitative et d'identifier des patterns de fonctionnements types et de moments clés en lien avec les émotions. Les accompagnateurs doivent être particulièrement vigilants dans ce repérage pour qu'à la fois les émotions positives perdurent et soutiennent l'engagement et que les émotions négatives ne soient pas uniquement synonymes de tensions et de baisse de motivation. 
En restant dans la portée pragmatique de ces projets destinés à professionnaliser, à développer des compétences, la question des émotions et particulièrement celles générées par le collectif est importante pour comprendre les dynamiques d'apprentissage et surtout pour faciliter ces dynamiques. Comprendre que des événements déclenchent des émotions qui, elles-mêmes, influencent la suite de l'activité et donc de l'apprentissage, permet d'outiller un dispositif pour limiter les impacts négatifs et capitaliser sur les émotions positives dès qu'un événement est identifié afin de soutenir la motivation. L'intention éducative sous-jacente à ces activités ou programmes est l'émancipation de nos étudiants. Apprendre à gérer ses émotions pour avancer avec le collectif fait partie de cet élan.

\section{Références bibliographiques}

Baumeister, R. F. (2016). Toward a general theory of motivation: Problems, challenges, opportunities, and the big picture. Motivation and Emotion, 40, 1-10.

Bornard, F. \& Briest-Breda, C. N. (2014). Développer l'esprit d'entreprendre, une question d'agilité. Revue de l'Entrepreneuriat, 13(2), 29-53.

Corbin, J. M. \& Strauss, A. (1990). Grounded theory research: Procedures, canons, and evaluative criteria. Qualitative sociology, 13(1), 3-21.

Corley, K. G. \& Gioia, D. A. (2004). Identity ambiguity and change in the wake of a corporate spin-off. Administrative science quarterly, 49(2), 173-208.

Côté, L. (2013/09). Améliorer ses stratégies de coping pour affronter le stress au travail. Psychologie Québec, dossier, 30(5), 41-44.

Csikszentmihalyi, M. (2002). Flow: The classic work on how to achieve happiness. London: Rider \& Co.

De Hoe, R. \& Janssen, F. (2014). L'échec entrepreneurial : une voie de succès futur. Le grand livre de l'économie PME, 177-195.

Deci, E. L. (1975). Intrinsic motivation. New York: Plenum Press.

Deci, E. L. \& Ryan, R. M. (1991). A motivational approach to self: Integration in personality. In R. A. Dienstbier (Ed.), Current theory and research in motivation, Vol. 38. Nebraska Symposium on Motivation, 1990: Perspectives on motivation (p. 237-288). University of Nebraska Press.

Deci, E. L. \& Ryan, R. M. (1985). Intrinsic Motivation and Self-Determination in Human Behavior. New York: Springer US.

Foliard, S. \& Le Pontois, S. (2017). Équipes entrepreneuriales étudiantes : comprendre pour agir. Entreprendre \& innover, (2), 44-54.

Gioia, D. A., Corley, K. G. \& Hamilton, A. L. (2013). Seeking qualitative rigor in inductive research: Notes on the Gioia methodology. Organizational research methods, 16(1), 15-31.

Günzel-Jensen, F. \& Robinson, S. (2017). Effectuation in the undergraduate classroom: three barriers to entrepreneurial learning. Education+ Training. 
Ionescu, S., Jacquet, M. M. \& Lhote, C. (1997). Les mécanismes de défense. Paris : Nathan Université.

Izard, C. E. (1993). Four systems for emotion activation: Cognitive and noncognitive processes. Psychological review, 100(1), 68.

Kyrö, P. (Ed.). (2015). Handbook of entrepreneurship and sustainable development research. Cheltenham: Edward Elgar Publishing.

Lazarus, R. S. \& Folkman, S. (1984). Coping and adaptation. In W. D. Gentry (Ed.), The Handbook of Behavioral Medicine (pp. 282-325), New York: Guilford.

Le Pontois, S. \& Jaillot, M. (2021, à paraître). Vive l'incertitude! Activer la créativité entrepreneuriale en générant des espaces tiers. Innovations.

Le Pontois, S. \& Foliard, S. (2018). Une vision à $360^{\circ}$ de l'accompagnement des équipes étudiantes. Entreprendre \& innover, (1), 55-66.

Michinov, N. \& Michinov, E. (2009). Investigating the relationship between transactive memory and performance in collaborative learning. Learning and Instruction, 19(1), 43-54.

Paulhan, I. (1992). Le concept de coping. L'année psychologique, 92(4), 545-557.

Pekrun, R. \& Frese, M. (1992). Emotions in work and achievement. International review of industrial and organizational psychology, 7, 153-200.

Piolat, A. \& Bannour, R. (2009). EMOTAIX : un scénario de Tropes pour l'identification automatisée du lexique émotionnel et affectif. L'Année psychologique, 109(4), 655-698.

Reeve, J. (2016). A grand theory of motivation: Why not? Motivation and Emotion, 40(1), 3135 .

Ryan, R. M. \& Deci, E. L. (2000). Intrinsic and extrinsic motivations: Classic definitions and new directions. Contemporary educational psychology, 25(1), 54-67.

Scherer, K. R. (2001). Appraisal considered as a process of multilevel sequential checking. Appraisal processes in emotion: Theory, methods, research, 92(120), 57.

Tcherkassof, A. \& Frijda, N. (2014). Les émotions : une conception relationnelle. L'Année psychologique, 114(3), 501-535.

Verzat, C. (2011). «Esprit d'entreprendre, es-tu là ? Mais de quoi parle-t-on ? Entreprendre \& Innover, 3, 7-18.

Verzat, C. \& O'Shea, N. (2019). Coopérer dans une équipe de projet entrepreneurial : une affaire de leadership ? Questions de Pédagogies dans l'Enseignement Supérieur, ENSTA Bretagne, IMT-A, UBO, Brest, France. 〈hal-02290511〉

Wegner, D. M. (1995). A computer network model of human transactive memory. Social cognition, 13(3), 319-339.

Woolley, A. W., Aggarwal, I. \& Malone, T. W. (2015). Collective intelligence and group performance. Current Directions in Psychological Science, 24(6), 420-424. 